\title{
A- Optimal Slope Design for Second Degree Kronecker Model Mixture Experiment With Four Ingredients With Application in Selected Fruits Blending
}

\author{
Ngigi Peter Kung'u ${ }^{1}$, J. K. Arap Koske ${ }^{2}$, Josphat K. Kinyanjui ${ }^{1}$ \\ ${ }^{1}$ Department of mathematics, statistics and actuarial science, Karatina University, Karatina, Kenya \\ 2 Department of mathematics \& computer science, Moi University, Eldoret, Kenya \\ Correspondence: Ngigi Peter Kung'u, Department of mathematics, statistics and actuarial science, Karatina University, \\ Karatina, Kenya
}

Received: December 13, 2020 Accepted: January 21, $2021 \quad$ Online Published: February 2, 2021

doi:10.5539/ijsp.v10n2p36

URL: https://doi.org/10.5539/ijsp.v10n2p36

\begin{abstract}
This study presents an investigation of an optimal slope design in the second degree Kronecker model for mixture experiments in four dimensions and its application in blending of selected fruits to prepare punch. The study centers around weighted centroid designs, with the second degree Kronecker model. This is guided by the fact that the class of weighted centroid designs is a complete class in the Kiefer Ordering. To overcome the problem of estimability, a concise coefficient matrix is defined that aid in selecting a maximal parameter subsystem for the Kronecker model. The information matrix of the design is obtained using a linear function of the moment matrices for the centroids and directly linked to the slope matrix. The discussion is based on Kronecker product algebra which clearly reflects the symmetries of the simplex experimental region. From the family of matrix means, a well-defined function is used to determine optimal values of the efficient developed design. Finally, a demonstration is provided for the case where the design is applied in fruit blending.
\end{abstract}

Keywords: information matrix, Kiefer ordering, moment matrix, optimal design, response surface methodology, slope, weighted centroid design

\section{Introduction}

This study explores the response surface with the intent of obtaining the optimal response. The response of interest is considered as a function of a set of independent factors. In this response surface methodology (RSM) problem we assume a response of interest is influenced by four factors with the intent of optimizing (obtaining maximal value of) this response. The response in linked to the factors through a second degree polynomial model.

In this mixture experiment the response is a function of the proportions of each ingredient. Let $x_{i}$ represent the proportion of the ith ingredient in the mixture. Then, factors involved are subject to two conditions, $x_{i} \geq 0, i=1,2,3,4$ and $\sum x_{i}=1$. The second constraint clearly shows that the levels of the four factors are interdependent. It is therefore easy to write a regression model without an intercept. The experimental region for this mixture problem is a three dimensional simplex.

\section{Materials and Methods}

Let $1_{\mathrm{m}}=(1, \ldots, 1)^{\prime} \in \mathfrak{R}^{m}$ be a unity vector. The experimental conditions $\mathrm{t}=\left(\mathrm{t}_{1}, \mathrm{t}_{2}, \ldots, \mathrm{t}_{\mathrm{m}}\right)$ with $\mathrm{t}_{\mathrm{i}} \geq 0$ of a mixture experiment are points in the probability simplex,

$$
T_{m}=\left\{t=\left(t_{1}, \quad t_{2}, \quad \ldots, t_{m}\right)^{\prime} \in[0, \quad 1]^{m}: 1_{m}{ }^{\prime} t=1\right\} .
$$

Under experimental conditions, $t \in T_{m}$, the response $Y_{t}$ is taken to be a quantitative random variable. The responses are assumed to be uncorrelated with equal but unknown finite variance say $\sigma^{2} \in(0, \infty)$. The design considered in this study has fifteen support points.

This study adopts Kronecker's second degree polynomial regression function with the expected response: 


$$
E\left(Y_{t}\right)=\sum_{i=1}^{m} \theta_{i i} t_{i}^{2}+\sum_{\substack{i, j=1 \\ i<j}}^{m}\left(\theta_{i j}+\theta_{j i}\right) t_{i} t_{j}
$$

where $Y_{t}$, is the response under experimental condition $t \in T_{m}$, and $\theta=\left(\theta_{11}, \theta_{12}, \ldots, \theta_{m m}\right) \in \mathfrak{R}^{m^{2}}$ an unknown parameter. (Draper, N. R. and Pukelsheim, F., 1998)

(Pukelsheim, 1993), gives a general review of the design environment. (Klein, 2004) and (Draper, N. R., Heiligers, B. and Pukelsheim, F., 2000), showed that the class of weighted centroid designs is a complete class for the Kiefer ordering.

\subsection{General Design Problem}

The problem of finding a design with maximum information on the parameter subsystem $K^{\prime} \theta$ can be formulated as;

$$
\begin{gathered}
\text { Maximize } \varphi_{p}\left(C_{k}(M(\tau))\right) \text { with } \tau \in T \\
\text { Subject to } C_{k}(M(\tau)) \in P D(s), \tau \in T
\end{gathered}
$$

where $\mathrm{T}$ denotes the set of all designs $\mathrm{T}_{\mathrm{m}}$. The side condition $C_{k}(M(\tau)) \in P D(s)$ is equal to the existence of an unbiased linear estimator for $K^{\prime} \theta$ under $\tau$, (Pukelsheim, 1993). In which case, the design $\tau$ is called feasible for $K^{\prime} \theta$. Any design solving problem (2) above for a fixed $\mathrm{p} \in(-\infty, 1]$ is said to be $\phi_{p}-$ optimal for $K^{\prime} \theta$ in $\mathrm{T}$. For all $\mathrm{p} \in(-\infty$, 1], (Pukelsheim, 1993) shows that $\phi_{p}$-optimal design for $K^{\prime} \theta$ exists.

\subsection{Moment Matrix}

An experimental design $\tau$ is a probability measure on the experimental domain with a finite number of support points. Each support point $s \in \sup p(\tau)$ directs the experimenter to take a proportion $\mathrm{T}(\{\mathrm{t}\})$ of all observations under experimental condition $\mathrm{T}$. The statistical properties of a design are reflected by its moment matrix:

$$
M(\tau)=\int_{\tau} f(t) f(t)^{\prime} d \tau \in N N D\left(m^{2}\right)
$$

where, $\mathrm{NND}\left(\mathrm{m}^{2}\right)$ denotes the cone of nonnegative definite $\mathrm{m}^{2} \times \mathrm{m}^{2}$ matrices. The entries of $\mathrm{M}(\tau)$ are fourth moments of $\tau$, since the regression function $f(t)$ is purely quadratic.

\subsection{Information Matrix}

We use unit vectors $\mathrm{e}_{1}, \mathrm{e}_{2}, \mathrm{e}_{3}, \mathrm{e}_{4}$ and set $e_{i j}=e_{i} \otimes e_{j}$ for $\mathrm{i}<\mathrm{j} \quad \mathrm{i}, \mathrm{j}=\{1,2,3,4\}$ and define the coefficient matrix

$$
K=\left(K_{1} ; K_{2}\right) \in \mathfrak{R}^{m^{2} \times\left(\begin{array}{c}
m+1 \\
2
\end{array}\right)}
$$

where

$$
K_{1}=\sum_{i=1}^{m} e_{i i} e_{i}^{\prime} \quad \text { and } K_{2}=\frac{1}{m} \sum_{\substack{i, j=1 \\ i<j}}^{m}\left(e_{i j}+e_{j i}\right) E_{i j}^{\prime}
$$

Obtainable as follows:

From, $e_{1}=\left(\begin{array}{lllll}1 & 0 & 0 & 0\end{array}\right)^{\prime}, e_{2}=\left(\begin{array}{lllll}0 & 1 & 0 & 0\end{array}\right)^{\prime} e_{3}=\left(\begin{array}{lllll}0 & 0 & 1 & 0\end{array}\right)^{\prime}$ and $e_{4}=\left(\begin{array}{lllll}0 & 0 & 0 & 0 & 1\end{array}\right)^{\prime}$ we have:

$$
\begin{aligned}
& e_{11}=e_{1} \otimes e_{1}=\left(\begin{array}{llllllllllllllll}
1 & 0 & 0 & 0 & 0 & 0 & 0 & 0 & 0 & 0 & 0 & 0 & 0 & 0 & 0 & 0
\end{array}\right)^{\prime}, \\
& e_{33}=e_{3} \otimes e_{3}=\left(\begin{array}{llllllllllllllll}
0 & 0 & 0 & 0 & 0 & 0 & 0 & 0 & 0 & 0 & 1 & 0 & 0 & 0 & 0 & 0
\end{array}\right)^{\prime} \text {, } \\
& e_{44}=e_{4} \otimes e_{4}=\left(\begin{array}{llllllllllllllll}
0 & 0 & 0 & 0 & 0 & 0 & 0 & 0 & 0 & 0 & 0 & 0 & 0 & 0 & 0 & 1
\end{array}\right)^{\prime} \text {, } \\
& e_{12}=e_{1} \otimes e_{2}=\left(\begin{array}{llllllllllllllll}
0 & 1 & 0 & 0 & 0 & 0 & 0 & 0 & 0 & 0 & 0 & 0 & 0 & 0 & 0 & 0
\end{array}\right)^{\prime} \\
& e_{21}=e_{2} \otimes e_{1}=\left(\begin{array}{llllllllllllllll}
0 & 0 & 0 & 0 & 1 & 0 & 0 & 0 & 0 & 0 & 0 & 0 & 0 & 0 & 0 & 0
\end{array}\right)^{\prime} \text {, } \\
& e_{13}=e_{1} \otimes e_{3}=\left(\begin{array}{llllllllllllllll}
0 & 0 & 1 & 0 & 0 & 0 & 0 & 0 & 0 & 0 & 0 & 0 & 0 & 0 & 0 & 0
\end{array}\right)^{\prime} \text {, } \\
& e_{31}=e_{3} \otimes e_{1}=\left(\begin{array}{llllllllllllllll}
0 & 0 & 0 & 0 & 0 & 0 & 0 & 0 & 1 & 0 & 0 & 0 & 0 & 0 & 0 & 0
\end{array}\right)^{\prime} \text {, }
\end{aligned}
$$




$$
\begin{aligned}
& e_{14}=e_{1} \otimes e_{4}=\left(\begin{array}{llllllllllllllll}
0 & 0 & 0 & 1 & 0 & 0 & 0 & 0 & 0 & 0 & 0 & 0 & 0 & 0 & 0 & 0
\end{array}\right)^{\prime} \\
& e_{41}=e_{4} \otimes e_{1}=\left(\begin{array}{llllllllllllllll}
0 & 0 & 0 & 0 & 0 & 0 & 0 & 0 & 0 & 0 & 0 & 0 & 1 & 0 & 0 & 0
\end{array}\right)^{\prime} \\
& e_{23}=e_{2} \otimes e_{3}=\left(\begin{array}{llllllllllllllll}
0 & 0 & 0 & 0 & 0 & 0 & 1 & 0 & 0 & 0 & 0 & 0 & 0 & 0 & 0 & 0
\end{array}\right)^{\prime}, \\
& e_{32}=e_{3} \otimes e_{2}=\left(\begin{array}{llllllllllllllll}
0 & 0 & 0 & 0 & 0 & 0 & 0 & 0 & 0 & 1 & 0 & 0 & 0 & 0 & 0 & 0
\end{array}\right)^{\prime}, \\
& e_{24}=e_{2} \otimes e_{4}=\left(\begin{array}{llllllllllllllll}
0 & 0 & 0 & 0 & 0 & 0 & 0 & 1 & 0 & 0 & 0 & 0 & 0 & 0 & 0 & 0
\end{array}\right)^{\prime}, \\
& e_{42}=e_{4} \otimes e_{2}=\left(\begin{array}{llllllllllllllll}
0 & 0 & 0 & 0 & 0 & 0 & 0 & 0 & 0 & 0 & 0 & 0 & 0 & 1 & 0 & 0
\end{array}\right)^{\prime}, \\
& e_{34}=e_{3} \otimes e_{4}=\left(\begin{array}{llllllllllllllll}
0 & 0 & 0 & 0 & 0 & 0 & 0 & 0 & 0 & 0 & 0 & 1 & 0 & 0 & 0 & 0
\end{array}\right)^{\prime} \text { and } \\
& e_{43}=e_{4} \otimes e_{3}=\left(\begin{array}{llllllllllllllll}
0 & 0 & 0 & 0 & 0 & 0 & 0 & 0 & 0 & 0 & 0 & 0 & 0 & 0 & 1 & 0
\end{array}\right)^{\prime}
\end{aligned}
$$

The vectors forming the standard basis of $\mathfrak{R}^{6}$ in a lexicographic order are:

$$
\begin{gathered}
E_{12}=\left(\begin{array}{llllll}
1 & 0 & 0 & 0 & 0 & 0
\end{array}\right)^{\prime}, \quad E_{13}=\left(\begin{array}{llllll}
0 & 1 & 0 & 0 & 0 & 0
\end{array}\right)^{\prime}, E_{14}=\left(\begin{array}{llllll}
0 & 0 & 1 & 0 & 0 & 0
\end{array}\right)^{\prime}, \\
E_{23}=\left(\begin{array}{llllll}
0 & 0 & 0 & 1 & 0 & 0
\end{array}\right)^{\prime}, E_{24}=\left(\begin{array}{lllllll}
0 & 0 & 0 & 0 & 1 & 0
\end{array}\right)^{\prime} \text { and } E_{34}=\left(\begin{array}{llllll}
0 & 0 & 0 & 0 & 0 & 1
\end{array}\right)^{\prime}
\end{gathered}
$$

Therefore, we obtain using (4);

$$
K_{1}=e_{11} e_{1}^{\prime}+e_{22} e_{2}^{\prime}+e_{33} e_{3}^{\prime}+e_{44} e_{4}^{\prime}=\left(\begin{array}{cccc}
1 & 0 & 0 & 0 \\
0 & 0 & 0 & 0 \\
0 & 0 & 0 & 0 \\
0 & 0 & 0 & 0 \\
0 & 0 & 0 & 0 \\
0 & 1 & 0 & 0 \\
0 & 0 & 0 & 0 \\
0 & 0 & 0 & 0 \\
0 & 0 & 0 & 0 \\
0 & 0 & 0 & 0 \\
0 & 0 & 1 & 0 \\
0 & 0 & 0 & 0 \\
0 & 0 & 0 & 0 \\
0 & 0 & 0 & 0 \\
0 & 0 & 0 & 0 \\
0 & 0 & 0 & 1
\end{array}\right)
$$

and

$$
\begin{gathered}
K_{2}=\frac{1}{4} \sum_{\substack{i, j=1 \\
i<j}}^{4}\left(e_{i j}+e_{j i}\right) E_{i j}^{\prime} \\
=\frac{1}{4}\left[\left(e_{12}+e_{21}\right) E_{12}^{\prime}+\left(e_{13}+e_{31}\right) E_{13}^{\prime}+\left(e_{14}+e_{41}\right) E_{14}^{\prime}+\left(e_{23}+e_{32}\right) E_{23}^{\prime}+\left(e_{24}+e_{42}\right) E_{24}^{\prime}+\left(e_{34}+e_{43}\right) E_{34}^{\prime}\right]
\end{gathered}
$$




$$
K_{2}=\left(\begin{array}{llllll}
0 & 0 & 0 & 0 & 0 & 0 \\
1 / 4 & 0 & 0 & 0 & 0 & 0 \\
0 & 1 / 4 & 0 & 0 & 0 & 0 \\
0 & 0 & 1 / 4 & 0 & 0 & 0 \\
1 / 4 & 0 & 0 & 0 & 0 & 0 \\
0 & 0 & 0 & 0 & 0 & 0 \\
0 & 0 & 0 & 1 / 4 & 0 & 0 \\
0 & 0 & 0 & 0 & 1 / 4 & 0 \\
0 & 1 / 4 & 0 & 0 & 0 & 0 \\
0 & 0 & 0 & 1 / 4 & 0 & 0 \\
0 & 0 & 0 & 0 & 0 & 0 \\
0 & 0 & 0 & 0 & 0 & 1 / 4 \\
0 & 0 & 1 / 4 & 0 & 0 & 0 \\
0 & 0 & 0 & 0 & 1 / 4 & 0 \\
0 & 0 & 0 & 0 & 0 & 1 / 4 \\
0 & 0 & 0 & 0 & 0 & 0
\end{array}\right)
$$

Thus

$$
K=\left(K_{1}, K_{2}\right)=\left(\begin{array}{llllllllll}
1 & 0 & 0 & 0 & 0 & 0 & 0 & 0 & 0 & 0 \\
0 & 0 & 0 & 0 & 1 / 4 & 0 & 0 & 0 & 0 & 0 \\
0 & 0 & 0 & 0 & 0 & 1 / 4 & 0 & 0 & 0 & 0 \\
0 & 0 & 0 & 0 & 0 & 0 & 1 / 4 & 0 & 0 & 0 \\
0 & 0 & 0 & 0 & 1 / 4 & 0 & 0 & 0 & 0 & 0 \\
0 & 1 & 0 & 0 & 0 & 0 & 0 & 0 & 0 & 0 \\
0 & 0 & 0 & 0 & 0 & 0 & 0 & 1 / 4 & 0 & 0 \\
0 & 0 & 0 & 0 & 0 & 0 & 0 & 0 & 1 / 4 & 0 \\
0 & 0 & 0 & 0 & 0 & 1 / 4 & 0 & 0 & 0 & 0 \\
0 & 0 & 0 & 0 & 0 & 0 & 0 & 1 / 4 & 0 & 0 \\
0 & 0 & 1 & 0 & 0 & 0 & 0 & 0 & 0 & 0 \\
0 & 0 & 0 & 0 & 0 & 0 & 0 & 0 & 0 & 1 / 4 \\
0 & 0 & 0 & 0 & 0 & 0 & 1 / 4 & 0 & 0 & 0 \\
0 & 0 & 0 & 0 & 0 & 0 & 0 & 0 & 1 / 4 & 0 \\
0 & 0 & 0 & 0 & 0 & 0 & 0 & 0 & 0 & 1 / 4 \\
0 & 0 & 0 & 1 & 0 & 0 & 0 & 0 & 0 & 0
\end{array}\right) .
$$

The full parameter vector $\theta \in \mathfrak{R}^{16}$ for model equation (1) is not estimable since the design matrix is column deficient. We select a maximal sub parameter vector:

$$
K^{\prime} \theta=\left\{\begin{array}{l}
\left(\theta_{i i}\right)_{1 \leq i \leq 4} \\
\frac{1}{4}\left(\theta_{i j}+\theta_{j i}\right)_{1 \leq i<j \leq 4}
\end{array}\right\} \in \mathfrak{R}^{10} \text { for all } \quad \theta \in \mathfrak{R}^{10}
$$

To optimize the response, we focus on the movement of the design center along the direction of the instantaneous slope of the response function, that is, $\frac{\partial Y_{t}}{\partial t}$. Designs attain certain properties in $\mathrm{Y}$ (estimated response) that do not coincide with the similar properties for the estimated slopes. Therefore, our focus is in experimental designs that are constructed based on derivatives (instantaneous changes), (Murty N. N. and Studden W. J., 1972) and (Ott L. S. and Mendenhall W., 1972).

(Aggarwal, M. L., Singh, P., Sarin, V. and Husain, B., 2013) obtained D-, A- and E-optimal orthogonal block designs for 
four mixture components in two blocks for Becker's models and K-model. This study investigates the slope of the response surface at a point $t$, over any specified direction. We develop the concept of robust slope over all directions. Define D, a matrix arising from the differentiation of $f(t)^{\prime} \theta$ with respect to each of the four independent factors, (Sung H. Park, Hyang S. Jung and Rabindra Nath Das, 2009). That is;

$$
D=\left(\frac{\partial f^{\prime}(t)}{\partial t_{1}} \frac{\partial f^{\prime}(t)}{\partial t_{2}}, \frac{\partial f^{\prime}(t)}{\partial t_{3}} \frac{\partial f^{\prime}(t)}{\partial t_{4}}\right)^{\prime}, \quad \text { where; } f(t)=t \otimes t \quad \text { and } t=\left(\begin{array}{llll}
t_{1} & t_{2} & t_{3} & t_{4}
\end{array}\right)^{\prime}
$$

An important matrix for the design with four ingredients is the adjusted $4 \times 10$ slope matrix $H_{0}=D K$.

The amount of information a design contains on $K^{\prime} \theta$ is captured by the information matrix:

$$
\mathrm{C}_{\mathrm{k}}(\mathrm{M}(\tau))=\min \left\{\mathrm{LM}(\tau) \mathrm{L}^{\prime} \quad \mathrm{L} \in \mathfrak{R}^{10 \times 16} ; \mathrm{LK}=I_{10}\right\}
$$

where $I_{10}$ denotes the $10 \times 10$ identity matrix and $\mathrm{L}$ is the left inverse of $\mathrm{K}$ derived from the computation, $L=\left(K^{\prime} K\right)^{-1} K^{\prime}$. The information matrix for $\mathrm{K}^{\prime} \theta$ which is a linear transformation of the moment matrix, takes the form:

$$
C_{0}=L M(\tau) L^{\prime} \in N N D(10)
$$

We then consider optimizing the information matrix for $\mathrm{K}^{\prime} \theta$ of the form:

$$
C=H_{0} C_{0} H_{0}^{\prime} \in N N N D(4)
$$

\subsection{Optimality Criteria}

We will compute optimal design for the Kronecker model using matrix mean $\phi_{p}$ which is an information function (Pukelsheim, 1993). For an information matrix $\mathrm{C}_{\mathrm{k}}(\mathrm{M}(\tau)) \in \mathrm{PD}(\mathrm{m})$ the kiefers $\phi_{p}$-criteria are defined by:

$$
\phi_{p}(C)=\left\{\begin{array}{ccc}
\lambda_{\text {min }}(C) \text { if } & p=-\infty \\
\operatorname{det}(C)^{\frac{1}{(m+1)}} \begin{array}{c}
2 \\
2
\end{array} & \text { if } & p=0 \\
{\left[\frac{1}{\left(\begin{array}{c}
m+1 \\
2
\end{array}\right)} \text { trace }^{p}\right]^{p} \text { if }} & p \in[-\infty ; 1] \backslash\{0\}
\end{array}\right.
$$

where $\lambda_{\text {min }}(\mathrm{C})$ refers to the smallest eigenvalue of $\mathrm{C}$. By definition $\phi_{p}(\mathrm{C})$ is a scalar measure which is a function of the eigenvalues of $\mathrm{C}$ for all $p \in[-\infty, 1]$. (Pukelsheim, 1993).

Consequently a design with maximum information on the parameter subsystem $K^{\prime} \theta$ solves the problem;

$$
\begin{gathered}
\text { Maximize } \phi_{p}\left(\mathrm{C}_{\mathrm{k}}(\mathrm{M}(\tau))\right) \text { with } \tau \in \mathrm{T} \\
\text { Subject to } \mathrm{C}_{\mathrm{k}}(\mathrm{M}(\tau)) \in \mathrm{PD}(\mathrm{m})
\end{gathered}
$$

Suppose $\eta(\alpha)$ satisfies the side condition $\mathrm{C}_{\mathrm{k}}(\mathrm{M}(\tau)) \in \mathrm{PD}(\mathrm{m})$ and write $\mathrm{C}_{\mathrm{j}}=\mathrm{C}_{\mathrm{k}}\left(\mathrm{M}\left(\eta_{j}\right)\right)$ for $\mathrm{j}=(1,2,3,4)$. For all $p \in(-\infty, 1], \eta(\alpha)$ solves problem (11) if and only if it satisfies the equivalence theorem;

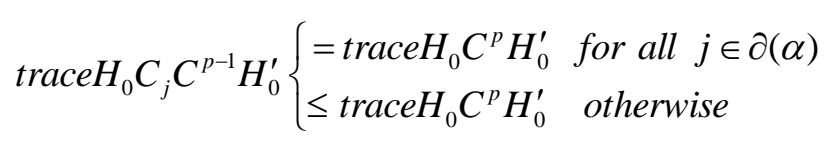

(Klein, 2004).

\section{Construction of the Design}

We consider the weighted centroid design $\eta(\alpha)=\sum_{j=1}^{4} \alpha_{j} \eta_{j}=\alpha_{1} \eta_{1}+\alpha_{2} \eta_{2}+\alpha_{3} \eta_{3}+\alpha_{4} \eta_{4}$ with four elementary centroids derived from support points: 
$\eta_{1}=\left\{\left(\begin{array}{l}1 \\ 0 \\ 0 \\ 0\end{array}\right),\left(\begin{array}{l}0 \\ 1 \\ 0 \\ 0\end{array}\right),\left(\begin{array}{l}0 \\ 0 \\ 1 \\ 0\end{array}\right),\left(\begin{array}{l}0 \\ 0 \\ 0 \\ 1\end{array}\right)\right\}, \quad \eta_{2}=\left\{\left(\begin{array}{l}1 / 2 \\ 1 / 2 \\ 0 \\ 0\end{array}\right),\left(\begin{array}{l}1 / 2 \\ 0 \\ 1 / 2 \\ 0\end{array}\right),\left(\begin{array}{l}1 / 2 \\ 0 \\ 0 \\ 1 / 2\end{array}\right),\left(\begin{array}{l}0 \\ 1 / 2 \\ 1 / 2 \\ 0\end{array}\right),\left(\begin{array}{l}0 \\ 1 / 2 \\ 0 \\ 1 / 2\end{array}\right),\left(\begin{array}{l}0 \\ 0 \\ 1 / 2 \\ 1 / 2\end{array}\right)\right\}, \quad \eta_{3}=\left\{\left(\begin{array}{l}1 / 3 \\ 1 / 3 \\ 1 / 3 \\ 0\end{array}\right),\left(\begin{array}{l}1 / 3 \\ 1 / 3 \\ 0 \\ 1 / 3\end{array}\right),\left(\begin{array}{l}1 / 3 \\ 0 \\ 1 / 3 \\ 1 / 3\end{array}\right),\left(\begin{array}{l}0 \\ 1 / 3 \\ 1 / 3 \\ 1 / 3\end{array}\right)\right\}$

and

$\eta_{4}=\left\{\left(\begin{array}{l}1 / 4 \\ 1 / 4 \\ 1 / 4 \\ 1 / 4\end{array}\right)\right\}$.

These weighted centroid designs discovered by (Sceffe', 1958) and (Sheffe'., 1963), are exchangeable and invariant under permutations, (Klein T. , 2004).

Thus the moment matrices for the two centroid designs $\eta_{1}$ and $\eta_{2}$ are:

$$
M\left(\eta_{1}\right)=\left(\begin{array}{cccccccccccccccc}
1 / 4 & 0 & 0 & 0 & 0 & 0 & 0 & 0 & 0 & 0 & 0 & 0 & 0 & 0 & 0 & 0 \\
0 & 0 & 0 & 0 & 0 & 0 & 0 & 0 & 0 & 0 & 0 & 0 & 0 & 0 & 0 & 0 \\
0 & 0 & 0 & 0 & 0 & 0 & 0 & 0 & 0 & 0 & 0 & 0 & 0 & 0 & 0 & 0 \\
0 & 0 & 0 & 0 & 0 & 0 & 0 & 0 & 0 & 0 & 0 & 0 & 0 & 0 & 0 & 0 \\
0 & 0 & 0 & 0 & 0 & 0 & 0 & 0 & 0 & 0 & 0 & 0 & 0 & 0 & 0 & 0 \\
0 & 0 & 0 & 0 & 0 & 1 / 4 & 0 & 0 & 0 & 0 & 0 & 0 & 0 & 0 & 0 & 0 \\
0 & 0 & 0 & 0 & 0 & 0 & 0 & 0 & 0 & 0 & 0 & 0 & 0 & 0 & 0 & 0 \\
0 & 0 & 0 & 0 & 0 & 0 & 0 & 0 & 0 & 0 & 0 & 0 & 0 & 0 & 0 & 0 \\
0 & 0 & 0 & 0 & 0 & 0 & 0 & 0 & 0 & 0 & 0 & 0 & 0 & 0 & 0 & 0 \\
0 & 0 & 0 & 0 & 0 & 0 & 0 & 0 & 0 & 0 & 0 & 0 & 0 & 0 & 0 & 0 \\
0 & 0 & 0 & 0 & 0 & 0 & 0 & 0 & 0 & 0 & 1 / 4 & 0 & 0 & 0 & 0 & 0 \\
0 & 0 & 0 & 0 & 0 & 0 & 0 & 0 & 0 & 0 & 0 & 0 & 0 & 0 & 0 & 0 \\
0 & 0 & 0 & 0 & 0 & 0 & 0 & 0 & 0 & 0 & 0 & 0 & 0 & 0 & 0 & 0 \\
0 & 0 & 0 & 0 & 0 & 0 & 0 & 0 & 0 & 0 & 0 & 0 & 0 & 0 & 0 & 0 \\
0 & 0 & 0 & 0 & 0 & 0 & 0 & 0 & 0 & 0 & 0 & 0 & 0 & 0 & 0 & 0 \\
0 & 0 & 0 & 0 & 0 & 0 & 0 & 0 & 0 & 0 & 0 & 0 & 0 & 0 & 0 & 1 / 4
\end{array}\right)
$$

and

$$
M\left(\eta_{2}\right)=\left(\begin{array}{cccccccccccccccc}
\frac{1}{32} & \frac{1}{96} & \frac{1}{96} & \frac{1}{96} & \frac{1}{96} & \frac{1}{96} & 0 & 0 & \frac{1}{96} & 0 & \frac{1}{96} & 0 & \frac{1}{96} & 0 & 0 & \frac{1}{96} \\
\frac{1}{96} & \frac{1}{96} & 0 & 0 & \frac{1}{96} & \frac{1}{96} & 0 & 0 & 0 & 0 & 0 & 0 & 0 & 0 & 0 & 0 \\
\frac{1}{96} & 0 & \frac{1}{96} & 0 & 0 & 0 & 0 & 0 & \frac{1}{96} & 0 & \frac{1}{96} & 0 & 0 & 0 & 0 & 0 \\
\frac{1}{96} & 0 & 0 & \frac{1}{96} & 0 & 0 & 0 & 0 & 0 & 0 & 0 & 0 & \frac{1}{96} & 0 & 0 & \frac{1}{96} \\
\frac{1}{96} & \frac{1}{96} & 0 & 0 & \frac{1}{96} & \frac{1}{96} & 0 & 0 & 0 & 0 & 0 & 0 & 0 & 0 & 0 & 0 \\
\frac{1}{96} & \frac{1}{96} & 0 & 0 & \frac{1}{96} & \frac{1}{32} & \frac{1}{96} & \frac{1}{96} & 0 & \frac{1}{96} & \frac{1}{96} & 0 & 0 & \frac{1}{96} & 0 & \frac{1}{96} \\
0 & 0 & 0 & 0 & 0 & \frac{1}{96} & \frac{1}{96} & 0 & 0 & \frac{1}{96} & \frac{1}{96} & 0 & 0 & 0 & 0 & 0 \\
0 & 0 & 0 & 0 & 0 & \frac{1}{96} & 0 & \frac{1}{96} & 0 & 0 & 0 & 0 & 0 & \frac{1}{96} & 0 & \frac{1}{96} \\
\frac{1}{96} & 0 & \frac{1}{96} & 0 & 0 & 0 & 0 & 0 & \frac{1}{96} & 0 & \frac{1}{96} & 0 & 0 & 0 & 0 & 0 \\
0 & 0 & 0 & 0 & 0 & \frac{1}{96} & \frac{1}{96} & 0 & 0 & \frac{1}{96} & \frac{1}{96} & 0 & 0 & 0 & 0 & 0 \\
\frac{1}{96} & 0 & \frac{1}{96} & 0 & 0 & \frac{1}{96} & \frac{1}{96} & 0 & \frac{1}{96} & \frac{1}{96} & \frac{1}{32} & \frac{1}{96} & 0 & 0 & \frac{1}{96} & \frac{1}{96} \\
0 & 0 & 0 & 0 & 0 & 0 & 0 & 0 & 0 & 0 & \frac{1}{96} & \frac{1}{96} & 0 & 0 & \frac{1}{96} & \frac{1}{96} \\
\frac{1}{96} & 0 & 0 & \frac{1}{96} & 0 & 0 & 0 & 0 & 0 & 0 & 0 & 0 & \frac{1}{96} & 0 & 0 & \frac{1}{96} \\
0 & 0 & 0 & 0 & 0 & \frac{1}{96} & 0 & \frac{1}{96} & 0 & 0 & 0 & 0 & 0 & \frac{1}{96} & 0 & \frac{1}{96} \\
0 & 0 & 0 & 0 & 0 & 0 & 0 & 0 & 0 & 0 & \frac{1}{96} & \frac{1}{96} & 0 & 0 & \frac{1}{96} & \frac{1}{96} \\
\frac{1}{96} & 0 & 0 & \frac{1}{96} & 0 & \frac{1}{96} & 0 & \frac{1}{96} & 0 & 0 & \frac{1}{96} & \frac{1}{96} & \frac{1}{96} & \frac{1}{96} & \frac{1}{96} & \frac{1}{32}
\end{array}\right)
$$

Defining the left inverse $\tilde{L}=\left(K^{\prime} K\right)^{-1} K^{\prime}$ where $\mathrm{K}$ is the coefficient matrix defined equation (4), 


$$
\tilde{L}=\left(K^{\prime} K\right)^{-1} K^{\prime}=\left(\begin{array}{cccccccccccccccc}
1 & 0 & 0 & 0 & 0 & 0 & 0 & 0 & 0 & 0 & 0 & 0 & 0 & 0 & 0 & 0 \\
0 & 0 & 0 & 0 & 0 & 1 & 0 & 0 & 0 & 0 & 0 & 0 & 0 & 0 & 0 & 0 \\
0 & 0 & 0 & 0 & 0 & 0 & 0 & 0 & 0 & 0 & 1 & 0 & 0 & 0 & 0 & 0 \\
0 & 0 & 0 & 0 & 0 & 0 & 0 & 0 & 0 & 0 & 0 & 0 & 0 & 0 & 0 & 1 \\
0 & 2 & 0 & 0 & 2 & 0 & 0 & 0 & 0 & 0 & 0 & 0 & 0 & 0 & 0 & 0 \\
0 & 0 & 2 & 0 & 0 & 0 & 0 & 0 & 2 & 0 & 0 & 0 & 0 & 0 & 0 & 0 \\
0 & 0 & 0 & 2 & 0 & 0 & 0 & 0 & 0 & 0 & 0 & 0 & 2 & 0 & 0 & 0 \\
0 & 0 & 0 & 0 & 0 & 0 & 2 & 0 & 0 & 2 & 0 & 0 & 0 & 0 & 0 & 0 \\
0 & 0 & 0 & 0 & 0 & 0 & 0 & 2 & 0 & 0 & 0 & 0 & 0 & 2 & 0 & 0 \\
0 & 0 & 0 & 0 & 0 & 0 & 0 & 0 & 0 & 0 & 0 & 2 & 0 & 0 & 2 & 0
\end{array}\right)
$$

The information matrices for the designs $\eta_{1}$ and $\eta_{2}$ are gotten as:

$$
C_{1}=C_{k}\left(M\left(\eta_{1}\right)\right)=\widetilde{L}\left(M\left(\eta_{1}\right)\right) \tilde{L}^{\prime}=\left(\begin{array}{cccccccccc}
1 / 4 & 0 & 0 & 0 & 0 & 0 & 0 & 0 & 0 & 0 \\
0 & 1 / 4 & 0 & 0 & 0 & 0 & 0 & 0 & 0 & 0 \\
0 & 0 & 1 / 4 & 0 & 0 & 0 & 0 & 0 & 0 & 0 \\
0 & 0 & 0 & 1 / 4 & 0 & 0 & 0 & 0 & 0 & 0 \\
0 & 0 & 0 & 0 & 0 & 0 & 0 & 0 & 0 & 0 \\
0 & 0 & 0 & 0 & 0 & 0 & 0 & 0 & 0 & 0 \\
0 & 0 & 0 & 0 & 0 & 0 & 0 & 0 & 0 & 0 \\
0 & 0 & 0 & 0 & 0 & 0 & 0 & 0 & 0 & 0 \\
0 & 0 & 0 & 0 & 0 & 0 & 0 & 0 & 0 & 0 \\
0 & 0 & 0 & 0 & 0 & 0 & 0 & 0 & 0 & 0
\end{array}\right)
$$

and

$$
C_{2}=C_{k}\left(M\left(\eta_{2}\right)\right)=\widetilde{L}\left(M\left(\eta_{2}\right)\right) \widetilde{L}^{\prime}=\left(\begin{array}{cccccccccc}
\frac{1}{32} & \frac{1}{96} & \frac{1}{96} & \frac{1}{96} & \frac{1}{24} & \frac{1}{24} & \frac{1}{24} & 0 & 0 & 0 \\
\frac{1}{96} & \frac{1}{32} & \frac{1}{96} & \frac{1}{96} & \frac{1}{24} & 0 & 0 & \frac{1}{24} & \frac{1}{24} & 0 \\
\frac{1}{96} & \frac{1}{96} & \frac{1}{32} & \frac{1}{96} & 0 & \frac{1}{24} & 0 & \frac{1}{24} & 0 & \frac{1}{24} \\
\frac{1}{96} & \frac{1}{96} & \frac{1}{96} & \frac{1}{32} & 0 & 0 & \frac{1}{24} & 0 & \frac{1}{24} & \frac{1}{24} \\
\frac{1}{24} & \frac{1}{24} & 0 & 0 & \frac{1}{6} & 0 & 0 & 0 & 0 & 0 \\
\frac{1}{24} & 0 & \frac{1}{24} & 0 & 0 & \frac{1}{6} & 0 & 0 & 0 & 0 \\
\frac{1}{24} & 0 & 0 & \frac{1}{24} & 0 & 0 & \frac{1}{6} & 0 & 0 & 0 \\
0 & \frac{1}{24} & \frac{1}{24} & 0 & 0 & 0 & 0 & \frac{1}{6} & 0 & 0 \\
0 & \frac{1}{24} & 0 & \frac{1}{24} & 0 & 0 & 0 & 0 & \frac{1}{6} & 0 \\
0 & 0 & \frac{1}{24} & \frac{1}{24} & 0 & 0 & 0 & 0 & 0 & \frac{1}{6}
\end{array}\right)
$$

Using equations (13) and (14), we obtain the information matrix for the design $\eta(\alpha)$ from; $C_{k}(M(\eta(\alpha)))=\alpha_{1} C\left(M\left(\eta_{1}\right)\right)+\alpha_{2} C\left(M\left(\eta_{2}\right)\right)$, as 


$$
C_{0}=\left(\begin{array}{cccccccccc}
\frac{8 \alpha_{1}+\alpha_{2}}{32} & \frac{\alpha_{2}}{96} & \frac{\alpha_{2}}{96} & \frac{\alpha_{2}}{96} & \frac{\alpha_{2}}{24} & \frac{\alpha_{2}}{24} & \frac{\alpha_{2}}{24} & 0 & 0 & 0 \\
\frac{\alpha_{2}}{96} & \frac{8 \alpha_{1}+\alpha_{2}}{32} & \frac{\alpha_{2}}{96} & \frac{\alpha_{2}}{96} & \frac{\alpha_{2}}{24} & 0 & 0 & \frac{\alpha_{2}}{24} & \frac{\alpha_{2}}{24} & 0 \\
\frac{\alpha_{2}}{96} & \frac{\alpha_{2}}{96} & \frac{8 \alpha_{1}+\alpha_{2}}{32} & \frac{\alpha_{2}}{96} & 0 & \frac{\alpha_{2}}{24} & 0 & \frac{\alpha_{2}}{24} & 0 & \frac{\alpha_{2}}{24} \\
\frac{\alpha_{2}}{96} & \frac{\alpha_{2}}{96} & \frac{\alpha_{2}}{96} & \frac{8 \alpha_{1}+\alpha_{2}}{32} & 0 & 0 & \frac{\alpha_{2}}{24} & 0 & \frac{\alpha_{2}}{24} & \frac{\alpha_{2}}{24} \\
\frac{\alpha_{2}}{24} & \frac{\alpha_{2}}{24} & 0 & 0 & \frac{\alpha_{2}}{6} & 0 & 0 & 0 & 0 & 0 \\
\frac{\alpha_{2}}{24} & 0 & \frac{\alpha_{2}}{24} & 0 & 0 & \frac{\alpha_{2}}{6} & 0 & 0 & 0 & 0 \\
\frac{\alpha_{2}}{24} & 0 & 0 & \frac{\alpha_{2}}{24} & 0 & 0 & \frac{\alpha}{6} & 0 & 0 & 0 \\
0 & \frac{\alpha_{2}}{24} & \frac{\alpha_{2}}{24} & 0 & 0 & 0 & 0 & \frac{\alpha_{2}}{6} & 0 & 0 \\
0 & \frac{\alpha_{2}}{24} & 0 & \frac{\alpha_{2}}{24} & 0 & 0 & 0 & 0 & \frac{\alpha_{2}}{6} & 0 \\
0 & 0 & \frac{\alpha_{2}}{24} & \frac{\alpha_{2}}{24} & 0 & 0 & 0 & 0 & 0 & \frac{\alpha_{2}}{6}
\end{array}\right)
$$

This matrix has an inverse,

$$
C_{0}^{-1}=\left(\begin{array}{cccccccccc}
\frac{4}{\alpha_{1}} & 0 & 0 & 0 & \frac{-1}{\alpha_{1}} & \frac{-1}{\alpha_{1}} & \frac{-1}{\alpha_{1}} & 0 & 0 & 0 \\
0 & \frac{4}{\alpha_{1}} & 0 & 0 & \frac{-1}{\alpha_{1}} & 0 & 0 & \frac{-1}{\alpha_{1}} & \frac{-1}{\alpha_{1}} & 0 \\
0 & 0 & \frac{4}{\alpha_{1}} & 0 & 0 & \frac{-1}{\alpha_{1}} & 0 & \frac{-1}{\alpha_{1}} & 0 & \frac{-1}{\alpha_{1}} \\
0 & 0 & 0 & \frac{4}{\alpha_{1}} & 0 & 0 & \frac{-1}{\alpha_{1}} & 0 & \frac{-1}{\alpha_{1}} & \frac{-1}{\alpha_{1}} \\
\frac{-1}{\alpha_{1}} & \frac{-1}{\alpha_{1}} & 0 & 0 & \frac{12 \alpha_{1}+\alpha_{2}}{2 \alpha_{1} \alpha_{2}} & \frac{1}{4 \alpha_{1}} & \frac{1}{4 \alpha_{1}} & \frac{1}{4 \alpha_{1}} & \frac{1}{4 \alpha_{1}} & 0 \\
\frac{-1}{\alpha_{1}} & 0 & \frac{-1}{\alpha_{1}} & 0 & \frac{1}{4 \alpha_{1}} & \frac{12 \alpha_{1}+\alpha_{2}}{2 \alpha_{1} \alpha_{2}} & \frac{1}{4 \alpha_{1}} & \frac{1}{4 \alpha_{1}} & 0 & \frac{1}{4 \alpha_{1}} \\
\frac{-1}{\alpha_{1}} & 0 & 0 & \frac{-1}{\alpha_{1}} & \frac{1}{4 \alpha_{1}} & \frac{1}{4 \alpha_{1}} & \frac{12 \alpha_{1}+\alpha_{2}}{2 \alpha_{1} \alpha_{2}} & 0 & \frac{1}{4 \alpha_{1}} & \frac{1}{4 \alpha_{1}} \\
0 & \frac{-1}{\alpha_{1}} & \frac{-1}{\alpha_{1}} & 0 & \frac{1}{4 \alpha_{1}} & \frac{1}{4 \alpha_{1}} & 0 & \frac{12 \alpha_{1}+\alpha_{2}}{2 \alpha_{1} \alpha_{2}} & \frac{1}{4 \alpha_{1}} & \frac{1}{4 \alpha_{1}} \\
0 & \frac{-1}{\alpha_{1}} & 0 & \frac{-1}{\alpha_{1}} & \frac{1}{4 \alpha_{1}} & 0 & \frac{1}{4 \alpha_{1}} & \frac{1}{4 \alpha_{1}} & \frac{12 \alpha_{1}+\alpha_{2}}{2 \alpha_{1} \alpha_{2}} & \frac{1}{4 \alpha_{1}} \\
0 & 0 & \frac{-1}{\alpha_{1}} & \frac{-1}{\alpha_{1}} & 0 & \frac{1}{4 \alpha_{1}} & \frac{1}{4 \alpha_{1}} & \frac{1}{4 \alpha_{1}} & \frac{1}{4 \alpha_{1}} & \frac{12 \alpha_{1}+\alpha_{2}}{2 \alpha_{1} \alpha_{2}}
\end{array}\right)
$$

which on squaring yields,

$$
C_{0}^{-2}=\left(\begin{array}{llllllllll}
a & b & b & b & c & c & c & d & d & d \\
b & a & b & b & c & d & d & c & c & d \\
b & b & a & b & d & c & d & c & d & c \\
b & b & b & a & d & d & c & d & c & c \\
c & c & d & d & e & f & f & f & f & g \\
c & d & c & d & f & e & f & f & g & f \\
c & d & d & c & f & f & e & g & f & f \\
d & c & c & d & f & f & g & e & f & f \\
d & c & d & c & f & g & f & f & e & f \\
d & d & c & c & g & f & f & f & f & e
\end{array}\right)
$$

where: 


$$
\begin{gathered}
a=\frac{19}{\alpha_{1}^{2}}, b=\frac{1}{\alpha_{1}^{2}}, c=\frac{-\left(6 \alpha_{1}+5 \alpha_{2}\right)}{\alpha_{1}^{2} \alpha_{2}}, d=\frac{-1}{2 \alpha_{1}^{2}}, e=\frac{65 \alpha_{1}^{2}+2 \alpha_{1}+5}{2 \alpha_{1}^{2} \alpha_{2}^{2}}, \\
f=\frac{24 \alpha_{1}+11 \alpha_{2}}{8 \alpha_{1}^{2} \alpha_{2}} \text { and } g=\frac{1}{4 \alpha_{1}^{2}}
\end{gathered}
$$

The slope matrix D as defined by equation (6) is obtained as

$$
D=\left(\begin{array}{cccccccccccccccc}
2 t_{1} & t_{2} & t_{3} & t_{4} & t_{2} & 0 & 0 & 0 & t_{3} & 0 & 0 & 0 & t_{4} & 0 & 0 & 0 \\
0 & t_{1} & 0 & 0 & t_{1} & 2 t_{2} & t_{3} & t_{4} & 0 & t_{3} & 0 & 0 & 0 & t_{4} & 0 & 0 \\
0 & 0 & t_{1} & 0 & 0 & 0 & t_{2} & 0 & t_{1} & t_{2} & 2 t_{3} & t_{4} & 0 & 0 & t_{4} & 0 \\
0 & 0 & 0 & t_{4} & 0 & 0 & 0 & t_{2} & 0 & 0 & 0 & t_{3} & t_{1} & t_{2} & t_{3} & 2 t_{4}
\end{array}\right)
$$

A corresponding adjusted slope matrix $H_{0}=D K$ is thus given by;

$$
H_{0}=\left(\begin{array}{cccccccccc}
2 t_{1} & 0 & 0 & 0 & \frac{1}{2} t_{2} & \frac{1}{2} t_{3} & \frac{1}{2} t_{4} & 0 & 0 & 0 \\
0 & 2 t_{2} & 0 & 0 & \frac{1}{2} t_{1} & 0 & 0 & \frac{1}{2} t_{3} & \frac{1}{2} t_{4} & 0 \\
0 & 0 & 2 t_{3} & 0 & 0 & \frac{1}{2} t_{1} & 0 & \frac{1}{2} t_{2} & 0 & \frac{1}{2} t_{4} \\
0 & 0 & 0 & 2 t_{4} & 0 & 0 & \frac{1}{2} t_{1} & 0 & \frac{1}{2} t_{2} & \frac{1}{2} t_{3}
\end{array}\right)
$$

To get the A-optimal design we invoke the equivalence theorem, that $\eta(\alpha)$ is $\phi_{p}$-optimal for $K^{\prime} \theta$ in T if and only if;

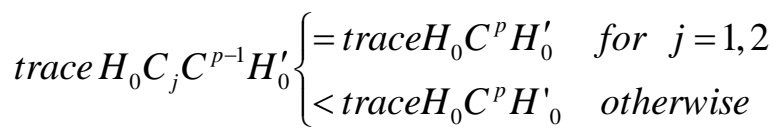

From which, the unique A-optimal design for $K^{\prime} \theta$ is arrived at when we substitute putting $\mathrm{p}=-1$.

$$
\text { trace }_{0} C_{j} C_{k}^{-2} H_{0}^{\prime}=\text { trace }_{0} C_{k}^{-1} H_{0}^{\prime}
$$

The following results were a consequence of using condition (17):

For $\mathrm{j}=1$ we have:

$$
\begin{aligned}
H_{0} C_{1} C_{o}^{-2} H_{0}^{\prime}= & \frac{1}{4 \alpha_{1}^{2}}\left[\begin{array}{lll}
76 t_{1}^{2}+A\left(t_{1} t_{2}+t_{1} t_{3}+t_{1} t_{4}\right) & 4 t_{1} t_{2}+A t_{1}^{2}-\frac{1}{2}\left(t_{1} t_{3}+t_{1} t_{4}\right) & 4 t_{1} t_{3}+A t_{1}^{2}-\frac{1}{2}\left(t_{1} t_{2}+t_{1} t_{4}\right) \\
4 t_{1} t_{2}+A t_{2}^{2}-\frac{1}{2}\left(t_{2} t_{3}+t_{2} t_{4}\right) & 76 t_{2}^{2}+A\left(t_{1} t_{2}+t_{2} t_{3}+t_{2} t_{4}\right) & 4 t_{2} t_{3}+A t_{2}^{2}-\frac{1}{2}\left(t_{1} t_{2}+t_{2} t_{4}\right) \\
4 t_{1} t_{3}+A t_{3}^{2}-\frac{1}{2}\left(t_{2} t_{3}+t_{3} t_{4}\right) & 4 t_{2} t_{3}+A t_{3}^{2}-\frac{1}{2}\left(t_{1} t_{3}+t_{3} t_{4}\right) & 76 t_{3}^{2}+A\left(t_{1} t_{3}+t_{2} t_{3}+t_{3} t_{4}\right) \\
4 t_{1} t_{4}+A t_{4}^{2}-\frac{1}{2}\left(t_{2} t_{4}+t_{3} t_{4}\right) & 4 t_{2} t_{4}+A t_{4}^{2}-\frac{1}{2}\left(t_{1} t_{4}+t_{3} t_{4}\right) & 4 t_{3} t_{4}+A t_{4}^{2}-\frac{1}{2}\left(t_{1} t_{4}+t_{2} t_{4}\right) \\
& 4 t_{1} t_{4}+A t_{1}^{2}-\frac{1}{2}\left(t_{1} t_{2}+t_{1} t_{3}\right) \\
& 4 t_{2} t_{4}+A t_{2}^{2}-\frac{1}{2}\left(t_{1} t_{2}+t_{2} t_{3}\right) \\
& 4 t_{3} t_{4}+A t_{3}^{2}-\frac{1}{2}\left(t_{1} t_{3}+t_{2} t_{3}\right) \\
& 76 t_{4}^{2}+A\left(t_{1} t_{4}+t_{2} t_{4}+t_{3} t_{4}\right)
\end{array}\right]
\end{aligned}
$$

where:

$$
A=\frac{-\left(6 \alpha_{1}+5 \alpha_{2}\right)}{\alpha_{2}}, t_{i}^{2}=\frac{103}{48}, i=1,2,3,4 \text { and } t_{i} t_{j}=\frac{77}{144}, i \neq j=1,2,3,4
$$

The trace being;

$$
\begin{aligned}
\text { trace }_{0} C_{1} C_{0}^{-2} H_{0}^{\prime}= & \frac{1}{4 \alpha_{1}^{2}}\left[76\left(t_{1}^{2}+t_{2}^{2}+t_{3}^{2}+t_{4}^{2}\right)-\frac{2\left(6 \alpha_{1}+5 \alpha_{2}\right)}{\alpha_{2}}\left(t_{1} t_{2}+t_{1} t_{3}+t_{1} t_{4}+t_{2} t_{3}+t_{2} t_{4}+t_{3} t_{4}\right)\right] \\
& =\frac{7443 \alpha_{2}-462 \alpha_{1}}{48 \alpha_{1}^{2} \alpha_{2}}
\end{aligned}
$$

and 


$$
\begin{aligned}
& H_{0} C_{0}^{-1} H_{0}^{\prime}=\frac{-1}{\alpha_{1}}\left[\begin{array}{c}
-16 t_{1}^{2}+\frac{a}{4}\left(t_{2}^{2}+t_{3}^{2}+t_{4}^{2}\right)+2\left(t_{1} t_{2}+t_{1} t_{3}+t_{1} t_{4}\right)-\frac{1}{8}\left(t_{2} t_{3}+t_{2} t_{4}+t_{3} t_{4}\right) \\
t_{2}^{2}+t_{1}^{2}-\frac{1}{16}\left(t_{3}^{2}+t_{4}^{2}+t_{1} t_{3}+t_{1} t_{4}+t_{2} t_{3}+t_{3} t_{4}\right)+\frac{a}{4} t_{1} t_{2} \\
t_{3}^{2}+t_{1}^{2}-\frac{1}{16}\left(t_{2}^{2}+t_{4}^{2}+t_{1} t_{2}+t_{1} t_{4}+t_{2} t_{3}+t_{3} t_{4}\right)+\frac{a}{4} t_{1} t_{3} \\
t_{4}^{2}+t_{1}^{2}-\frac{1}{16}\left(t_{2}^{2}+t_{3}^{2}+t_{1} t_{2}+t_{1} t_{3}+t_{2} t_{4}+t_{3} t_{4}\right)+\frac{a}{4} t_{1} t_{4}
\end{array}\right. \\
& t_{1}^{2}+t_{2}^{2}-\frac{1}{16}\left(t_{3}^{2}+t_{4}^{2}+t_{1} t_{3}+t_{1} t_{4}+t_{2} t_{3}+t_{2} t_{4}\right)+\frac{a}{4} t_{1} t_{2} \\
& -16 t_{2}^{2}+\frac{a}{4}\left(t_{1}^{2}+t_{3}^{2}+t_{4}^{2}\right)+2\left(t_{1} t_{2}+t_{2} t_{3}+t_{2} t_{4}\right)-\frac{1}{8}\left(t_{1} t_{3}+t_{1} t_{4}+t_{3} t_{4}\right) \\
& t_{3}^{2}+t_{2}^{2}-\frac{1}{16}\left(t_{1}^{2}+t_{4}^{2}+t_{1} t_{2}+t_{1} t_{3}+t_{2} t_{4}+t_{3} t_{4}\right)+\frac{a}{4} t_{2} t_{3} \\
& t_{4}^{2}+t_{2}^{2}-\frac{1}{16}\left(t_{1}^{2}+t_{3}^{2}+t_{1} t_{2}+t_{1} t_{4}+t_{2} t_{3}+t_{3} t_{4}\right)+\frac{a}{4} t_{2} t_{4} \\
& t_{1}^{2}+t_{3}^{2}-\frac{1}{16}\left(t_{2}^{2}+t_{4}^{2}+t_{1} t_{2}+t_{1} t_{4}+t_{2} t_{3}+t_{3} t_{4}\right)+\frac{a}{4} t_{1} t_{3} \\
& t_{2}^{2}+t_{3}^{2}-\frac{1}{16}\left(t_{1}^{2}+t_{4}^{2}+t_{1} t_{2}+t_{1} t_{3}+t_{2} t_{4}+t_{3} t_{4}\right)+\frac{a}{4} t_{2} t_{3} \\
& -16 t_{3}^{2}+\frac{a}{4}\left(t_{1}^{2}+t_{2}^{2}+t_{4}^{2}\right)+2\left(t_{1} t_{3}+t_{2} t_{3}+t_{3} t_{4}\right)-\frac{1}{8}\left(t_{1} t_{2}+t_{1} t_{4}+t_{2} t_{4}\right) \\
& t_{4}^{2}+t_{3}^{2}-\frac{1}{16}\left(t_{1}^{2}+t_{2}^{2}+t_{1} t_{3}+t_{1} t_{4}+t_{2} t_{3}+t_{2} t_{4}\right)+\frac{a}{4} t_{3} t_{4} \\
& t_{1}^{2}+t_{4}^{2}-\frac{1}{16}\left(t_{2}^{2}+t_{3}^{2}+t_{1} t_{2}+t_{1} t_{3}+t_{2} t_{4}+t_{3} t_{4}\right)+\frac{a}{4} t_{1} t_{4} \\
& t_{2}^{2}+t_{4}^{2}-\frac{1}{16}\left(t_{1}^{2}+t_{3}^{2}+t_{1} t_{2}+t_{1} t_{4}+t_{2} t_{3}+t_{3} t_{4}\right)+\frac{a}{4} t_{2} t_{4} \\
& t_{3}^{2}+t_{4}^{2}-\frac{1}{16}\left(t_{1}^{2}+t_{2}^{2}+t_{1} t_{3}+t_{1} t_{4}+t_{2} t_{3}+t_{2} t_{4}\right)+\frac{a}{4} t_{3} t_{4} \\
& \left.-16 t_{4}^{2}+\frac{a}{4}\left(t_{1}^{2}+t_{2}^{2}+t_{3}^{2}\right)+2\left(t_{1} t_{4}+t_{2} t_{4}+t_{3} t_{4}\right)-\frac{1}{8}\left(t_{1} t_{2}+t_{1} t_{3}+t_{2} t_{3}\right)\right]
\end{aligned}
$$

where:

$$
a=\frac{-\left(12 \alpha_{1}+\alpha_{2}\right)}{2 \alpha_{2}}, t_{i}^{2}=\frac{103}{48}, i=1,2,3,4 \text { and } t_{i} t_{j}=\frac{77}{144}, i \neq j=1,2,3,4
$$

Trace of this matrix is;

$$
\begin{aligned}
\operatorname{trace~}_{0} C_{k}^{-1} H_{0}^{\prime} & =\frac{-1}{\alpha_{1}}\left[\left(-16+\frac{3}{4} a\right)\left(t_{1}^{2}+t_{2}^{2}+t_{3}^{2}+t_{4}^{2}\right)+\frac{15}{4}\left(t_{1} t_{2}+t_{1} t_{3}+t_{1} t_{4}+t_{2} t_{3}+t_{2} t_{4}+t_{3} t_{4}\right)\right] \\
& =\frac{1854 \alpha_{1}+6169 \alpha_{2}}{48 \alpha_{1} \alpha_{2}}
\end{aligned}
$$

From condition (17) using the values from (19) and (20) we have;

$$
\frac{7443 \alpha_{2}-462 \alpha_{1}}{48 \alpha_{1}^{2} \alpha_{2}}=\frac{1854 \alpha_{1}+6169 \alpha_{2}}{48 \alpha_{1} \alpha_{2}}
$$

giving a plausible value $\alpha_{1}=0.664039581$.

For $\mathrm{j}=2$;

The following elementary evaluation are realized: 


$$
\begin{aligned}
& H_{0} C_{2} C_{0}^{-1} H_{0}^{\prime}=\frac{-1}{4 \alpha_{1} \alpha_{2}}\left[\begin{array}{c}
12 t_{1}^{2}+\frac{1}{4} d\left(t_{2}^{2}+t_{3}^{2}+t_{4}^{2}\right)+(4+a) t_{1}\left(t_{2}+t_{3}+t_{4}\right)-\frac{1}{2}\left(t_{2} t_{3}+t_{2} t_{4}+t_{3} t_{4}\right) \\
4 t_{1}\left(t_{1}+t_{2}\right)+2 t_{2}\left(2 a t_{2}-t_{3}-t_{4}\right)+\frac{1}{4} t_{1}\left(d t_{2}-t_{3}-t_{4}\right)-\frac{1}{4} t_{3}\left(t_{2}+t_{3}\right)-\frac{1}{4} t_{4}\left(t_{2}+t_{4}\right) \\
4 t_{1}\left(t_{1}+t_{3}\right)+2 t_{3}\left(2 a t_{3}-t_{2}-t_{4}\right)+\frac{1}{4} t_{1}\left(d t_{3}-t_{2}-t_{4}\right)-\frac{1}{4} t_{2}\left(t_{2}+t_{3}\right)-\frac{1}{4} t_{4}\left(t_{3}+t_{4}\right) \\
4 t_{1}\left(t_{1}+t_{4}\right)+2 t_{4}\left(2 a t_{4}-t_{2}-t_{3}\right)+\frac{1}{4} t_{1}\left(d t_{4}-t_{2}-t_{3}\right)-\frac{1}{4} t_{2}\left(t_{2}+t_{4}\right)-\frac{1}{4} t_{3}\left(t_{3}+t_{4}\right)
\end{array}\right. \\
& 4 t_{2}\left(t_{2}+t_{1}\right)+2 t_{1}\left(2 a t_{1}-t_{3}-t_{4}\right)+\frac{1}{4} t_{2}\left(d t_{1}-t_{3}-t_{4}\right)-\frac{1}{4} t_{3}\left(t_{1}+t_{3}\right)-\frac{1}{4} t_{4}\left(t_{1}+t_{4}\right) \\
& 12 t_{2}^{2}+\frac{1}{4} d\left(t_{1}^{2}+t_{3}^{2}+t_{4}^{2}\right)+(4+a) t_{2}\left(t_{1}+t_{3}+t_{4}\right)-\frac{1}{2}\left(t_{1} t_{3}+t_{1} t_{4}+t_{3} t_{4}\right) \\
& 4 t_{2}\left(t_{2}+t_{3}\right)+2 t_{3}\left(2 a t_{3}-t_{1}-t_{4}\right)+\frac{1}{4} t_{2}\left(d t_{3}-t_{1}-t_{4}\right)-\frac{1}{4} t_{1}\left(t_{1}+t_{3}\right)-\frac{1}{4} t_{4}\left(t_{3}+t_{4}\right) \\
& 4 t_{2}\left(t_{2}+t_{4}\right)+2 t_{4}\left(2 a t_{4}-t_{1}-t_{3}\right)+\frac{1}{4} t_{2}\left(d t_{4}-t_{1}-t_{3}\right)-\frac{1}{4} t_{1}\left(t_{1}+t_{4}\right)-\frac{1}{4} t_{3}\left(t_{3}+t_{4}\right) \\
& 4 t_{3}\left(t_{3}+t_{1}\right)+2 t_{1}\left(2 a t_{1}-t_{2}-t_{4}\right)+\frac{1}{4} t_{3}\left(d t_{1}-t_{2}-t_{4}\right)-\frac{1}{4} t_{2}\left(t_{1}+t_{2}\right)-\frac{1}{4} t_{4}\left(t_{1}+t_{4}\right) \\
& 4 t_{3}\left(t_{3}+t_{2}\right)+2 t_{2}\left(2 a t_{2}-t_{1}-t_{4}\right)+\frac{1}{4} t_{3}\left(d t_{2}-t_{1}-t_{4}\right)-\frac{1}{4} t_{1}\left(t_{1}+t_{2}\right)-\frac{1}{4} t_{4}\left(t_{2}+t_{4}\right) \\
& 12 t_{3}^{2}+\frac{1}{4} d\left(t_{1}^{2}+t_{2}^{2}+t_{4}^{2}\right)+(4+a) t_{3}\left(t_{1}+t_{2}+t_{4}\right)-\frac{1}{2}\left(t_{1} t_{2}+t_{1} t_{4}+t_{2} t_{4}\right) \\
& 4 t_{3}\left(t_{3}+t_{4}\right)+2 t_{4}\left(2 a t_{4}-t_{1}-t_{2}\right)+\frac{1}{4} t_{3}\left(d t_{4}-t_{1}-t_{2}\right)-\frac{1}{4} t_{1}\left(t_{1}+t_{4}\right)-\frac{1}{4} t_{2}\left(t_{2}+t_{4}\right) \\
& 4 t_{4}\left(t_{4}+t_{1}\right)+2 t_{1}\left(2 a t_{1}-t_{2}-t_{3}\right)+\frac{1}{4} t_{4}\left(d t_{1}-t_{2}-t_{3}\right)-\frac{1}{4} t_{2}\left(t_{1}+t_{2}\right)-\frac{1}{4} t_{3}\left(t_{1}+t_{3}\right) \\
& 4 t_{4}\left(t_{4}+t_{2}\right)+2 t_{2}\left(2 a t_{2}-t_{1}-t_{3}\right)+\frac{1}{4} t_{4}\left(d t_{2}-t_{1}-t_{3}\right)-\frac{1}{4} t_{1}\left(t_{1}+t_{2}\right)-\frac{1}{4} t_{3}\left(t_{2}+t_{3}\right) \\
& 4 t_{4}\left(t_{4}+t_{3}\right)+2 t_{3}\left(2 a t_{3}-t_{1}-t_{2}\right)+\frac{1}{4} t_{4}\left(d t_{3}-t_{1}-t_{2}\right)-\frac{1}{4} t_{1}\left(t_{1}+t_{3}\right)-\frac{1}{4} t_{2}\left(t_{2}+t_{3}\right) \\
& 12 t_{4}^{2}+\frac{1}{4} d\left(t_{1}^{2}+t_{2}^{2}+t_{3}^{2}\right)+(4+a) t_{4}\left(t_{1}+t_{2}+t_{3}\right)-\frac{1}{2}\left(t_{1} t_{2}+t_{1} t_{3}+t_{2} t_{3}\right)
\end{aligned}
$$

where:

$$
a=\frac{-\left(5 \alpha_{1}+1\right)}{\alpha_{2}}, d=\frac{-2\left(11 \alpha_{1}+1\right)}{\alpha_{2}}, \quad t_{i}^{2}=\frac{103}{48}, i=1,2,3,4 \text { and } t_{i} t_{j}=\frac{77}{144}, i \neq j=1,2,3,4
$$

The trace of matrix being,

$$
\begin{aligned}
{\text { trace } H_{0} C_{2} C_{0}^{-2} H^{\prime}}^{\prime} & =\frac{-1}{4 \alpha_{1} \alpha_{2}}\left[\left(12+\frac{3}{4} d\right)\left(t_{1}^{2}+t_{2}^{2}+t_{3}^{2}+t_{4}^{2}\right)+(7+2 a)\left(t_{1} t_{2}+t_{1} t_{3}+t_{1} t_{4}+t_{2} t_{3}+t_{2} t_{4}+t_{3} t_{4}\right)\right] \\
& =\frac{463+4169 \alpha_{1}-3011 \alpha_{2}}{96 \alpha_{1} \alpha_{2}^{2}}
\end{aligned}
$$

From condition (17) and the values in (19) and (20) we have;

$$
\frac{1854 \alpha_{1}+6169 \alpha_{2}}{48 \alpha_{1} \alpha_{2}}=\frac{463+4169 \alpha_{1}-3011 \alpha_{2}}{96 \alpha_{1} \alpha_{2}^{2}},
$$

with the unique solution $\alpha_{2}=0.335960419$.

Therefore the unique A-slope optimal design for $K^{\prime} \theta$ is

$$
\eta\left(\alpha^{A}\right)=\alpha_{1} \eta_{1}+\alpha_{2} \eta_{2}=0.664039581 \eta_{1}+0.335960419 \eta_{2} .
$$

The information matrix after employing the ordinates of the support points and the optimal vector becomes:

$$
H_{0} C_{0}^{-1} H_{0}^{\prime}=\left[\begin{array}{cccc}
77.1282 & -3.3696 & -3.3696 & -3.3696 \\
-3.3696 & 77.1282 & -3.3696 & -3.3696 \\
-3.3696 & -3.3696 & 77.1282 & -3.3696 \\
-3.3696 & -3.3696 & -3.3696 & 77.1282
\end{array}\right]
$$

The maximum of the A-criterion using (10) is given by; 


$$
v\left(\phi_{-1}\right)=\left(\frac{1}{4} \text { trace }_{0} C_{0}^{-1} H_{0}^{\prime}\right)^{-1}=(77.1282)^{-1}=0.012965426 .
$$

\section{Fruit Blending Experiment}

Four fruits (pine apple, pawpaw, banana and coconut) were involved in the experiment. The response on a scale 1-15 was taken as the average score for the four attributes considered: taste, colour, texture and smell. The sixty data values are from fifteen support points for the weighted centroid design each replicated four times. The points comprised the four pure blends, six binary blends, four ternary blend and the four fruits together in the mixture.

\subsection{Sample Data: For Four Replicates}

\begin{tabular}{|l|l|l|l|l|l|l|l|l|l|l|}
\hline \multicolumn{3}{|l}{ Combinations (support points) } & \multicolumn{4}{l|}{ ATTRIBUTES } & TOTAL & MEAN \\
\hline pina apple & paw paw & banana & coconut & texture & colour & taste & smell & & \\
\hline 1 & 0 & 0 & 0 & 10 & 8 & 10 & 12 & 40 & 10.00 \\
\hline 1 & 0 & 0 & 0 & 12 & 15 & 11 & 9 & 47 & 11.75 \\
\hline 1 & 0 & 0 & 0 & 8 & 9 & 11 & 11 & 39 & 9.75 \\
\hline 1 & 0 & 0 & 0 & 14 & 14 & 14 & 15 & 57 & 14.25 \\
\hline$\cdot$ &. &. &. &. &. &. &. &. &. \\
$\cdot$ &. &. &. &. &. &. &. &. &. \\
. &. &. &. &. &. &. &. &. &. \\
\hline 0.25 & 0.25 & 0.25 & 0.25 & 11 & 13 & 10 & 15 & 49 & 12.25 \\
\hline 0.25 & 0.25 & 0.25 & 0.25 & 9 & 8 & 11 & 10 & 38 & 9.50 \\
\hline 0.25 & 0.25 & 0.25 & 0.25 & 10 & 13 & 12 & 14 & 49 & 12.25 \\
\hline 0.25 & 0.25 & 0.25 & 0.25 & 9 & 7 & 10 & 13 & 39 & 9.75 \\
\hline
\end{tabular}

\subsection{Fitted Model}

The estimates of the coefficients for the Kronecker model were obtained using SAS software package. The model from equation (4) with $\mathrm{m}=4$ is;

$$
\begin{aligned}
\hat{y}=E(y)= & 11.17(\text { pineapple })^{2}+10.50(\text { pawpaw })^{2}+10.12(\text { banana })^{2}+9.31(\text { coconut })^{2}+ \\
& 24.68 \text { peneapple } * \text { pawpaw }+21.54 \text { pineapple } * \text { banana }+16.40 \text { pineapple } * \text { coconut }+ \\
& 19.25 \text { pawpaw } * \text { banana }+10.86 \text { pawpaw } * \text { coconut }+16.72 \text { banana } * \text { coconut }
\end{aligned}
$$

\subsection{Model Validity}

We performed the analysis on the model validity to examine the fitted model if it provides a good approximation of the true response surface. Analysis of variance (ANOVA) was used to examine the Kronecker model. As is evident from the output table 1 below, $96.67 \%$ of the variation in the response is accounted for by the purposeful changes made on the amounts of each fruit in the mixture. The overall model is highly significant with an estimated probability value less than 0.0001 , much lower than the 0.05 and 0.01 levels of significance.

Table 1. ANOVA for the four ingredients Kronecker model

The GLM Procedure

Dependent Variable: yield

\begin{tabular}{lccccc} 
& \multicolumn{2}{c}{ Sum of } & & & \\
Source & DF & Squares & Mean Square & F Value & $\operatorname{Pr}>$ F \\
Model & 10 & 5794.907380 & 579.490738 & 145.17 & $<.0001$ \\
Error & 50 & 199.592620 & 3.991852 & & \\
Uncorrected Total & & 60 & 5994.500000 & &
\end{tabular}

R-Square Coeff Var Root MSE yield Mean

$\begin{array}{llll}0.966704 & 20.43951 & 1.997962 & 9.775000\end{array}$ 
NOTE: No intercept term is used: R-square is not corrected for the mean.

We also used the t-test values to test for the significance of the individual coefficients (hence fruits) in the model. The test is, we test the null hypothesis $H_{o}: \beta_{i j}=0$ against the alternative hypothesis $H_{1}: \beta_{i j} \neq 0$. From the information below (in table 2), all the coefficients are significant with small estimated probability $(\operatorname{Pr}>|t|)$ values at the 0.05 level of significance. The interaction between pawpaw and coconut, compared to the other coefficients, is the least significant at the 0.01 level of significance.

Table 2. T-test Values for coefficients of the four ingredients Kronecker model

The GLM Procedure

Dependent Variable: yield

$\begin{array}{lcccc}\text { Standard } & & & \\ \text { Parameter } & \text { Estimate } & \text { Error } t \text { Value } & \operatorname{Pr}>|t| & \\ \text { pine*pine } & 11.16953443 & 0.98751632 & 11.31 & <.0001 \\ \text { paw*paw } & 10.49541136 & 0.98751632 & 10.63 & <.0001 \\ \text { ban*ban } & 10.12119869 & 0.98751632 & 10.25 & <.0001 \\ \text { coco*coco } & 9.30566494 & 0.98751632 & 9.42 & <.0001 \\ \text { pine*paw } & 24.67598681 & 3.63911375 & 6.78 & <.0001 \\ \text { pine*ban } & 21.53760215 & 3.63911375 & 5.92 & <.0001 \\ \text { pine*coco } & 16.40167588 & 3.63911375 & 4.51 & <.0001 \\ \text { paw*ban } & 19.24769445 & 3.63911375 & 5.29 & <.0001 \\ \text { paw*coco } & 10.86176818 & 3.63911375 & 2.98 & 0.0044 \\ \text { ban*coco } & 16.72338352 & 3.63911375 & 4.60 & <.0001\end{array}$

The assumption of normality on the errors, clearly points to a similar distribution on the observations. By examination of the P-P and Q-Q plots in figure 1 below, there is no indication of any serious deviation from normality.

Normal P-P Plot of RESPONSE

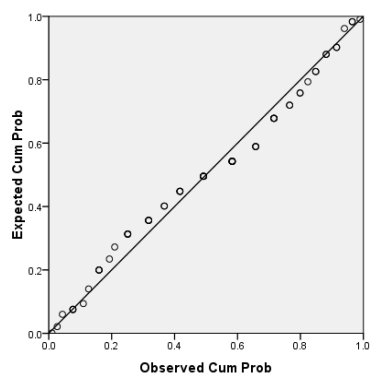

Normal Q-Q Plot of RESPONSE

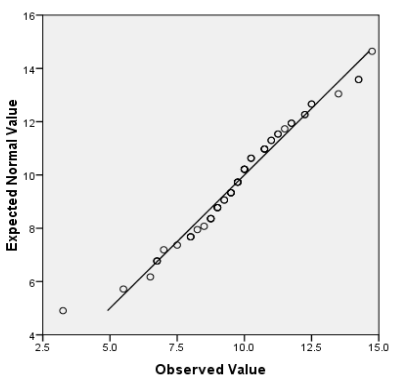

Figure 1.

\subsection{Slope Information for the A-optimal Criterion}

To get the A-optimal slope value for the design with the four ingredients, we first pre- and post-multiplied the inverse of information matrix (15) with the adjusted slope matrix $\left(\mathrm{H}_{0}\right)$, to get the necessary slope information matrix; 


$$
\begin{aligned}
& C=\left(\begin{array}{l}
\frac{16 a^{2}}{\alpha_{1}} t_{1}^{2}+\frac{\left(12 \alpha_{1}+\alpha_{2}\right)\left(e^{2} t_{2}^{2}+f^{2} t_{3}^{2}+g^{2} t_{4}^{2}\right)}{32 \alpha_{1} \alpha_{2}}-\frac{a t_{1}\left(e t_{2}+f t_{3}+g t_{4}\right)}{\alpha_{1}}+\frac{e f t_{2} t_{3}+e g t_{2} t_{4}+f g t_{3} t_{4}}{32 \alpha_{1}} \\
-\frac{b e}{2 \alpha_{1}} t_{2}^{2}-\frac{a e}{2 \alpha_{1}} t_{1}^{2}+\frac{f h}{64 \alpha_{1}} t_{3}^{2}+\frac{g k}{64 \alpha_{1}} t_{4}^{2}+\frac{e\left(f t_{1} t_{3}+g t_{1} t_{4}+h t_{2} t_{3}+k t_{2} t_{4}\right)}{64 \alpha_{1}}+\frac{\left(12 \alpha_{1}+\alpha_{2}\right) e^{2} t_{1} t_{2}}{32 \alpha_{1} \alpha_{2}} \\
-\frac{c f}{2 \alpha_{1}} t_{3}^{2}-\frac{a f}{2 \alpha_{1}} t_{1}^{2}+\frac{e h}{64 \alpha_{1}} t_{2}^{2}+\frac{g m}{64 \alpha_{1}} t_{4}^{2}+\frac{f\left(e t_{1} t_{2}+g t_{1} t_{4}+h t_{2} t_{3}+m t_{3} t_{4}\right)}{64 \alpha_{1}}+\frac{\left(12 \alpha_{1}+\alpha_{2}\right) f^{2} t_{1} t_{3}}{32 \alpha_{1} \alpha_{2}} \\
-\frac{d g}{2 \alpha_{1}} t_{4}^{2}-\frac{a g}{2 \alpha_{1}} t_{1}^{2}+\frac{e k}{64 \alpha_{1}} t_{2}^{2}+\frac{f m}{64 \alpha_{1}} t_{3}^{2}+\frac{g\left(e t_{1} t_{2}+f t_{1} t_{3}+k t_{2} t_{4}+m t_{3} t_{4}\right)}{64 \alpha_{1}}+\frac{\left(12 \alpha_{1}+\alpha_{2}\right) g^{2} t_{1} t_{4}}{32 \alpha_{1} \alpha_{2}}
\end{array}\right. \\
& -\frac{b e}{2 \alpha_{1}} t_{2}^{2}-\frac{a e}{2 \alpha_{1}} t_{1}^{2}+\frac{f h}{64 \alpha_{1}} t_{3}^{2}+\frac{g k}{64 \alpha_{1}} t_{4}^{2}+\frac{e\left(f t_{1} t_{3}+g t_{1} t_{4}+h t_{2} t_{3}+k t_{2} t_{4}\right)}{64 \alpha_{1}}+\frac{\left(12 \alpha_{1}+\alpha_{2}\right) e^{2} t_{1} t_{2}}{32 \alpha_{1} \alpha_{2}} \\
& \frac{16 b^{2}}{\alpha_{1}} t_{2}^{2}+\frac{\left(12 \alpha_{1}+\alpha_{2}\right)\left(e^{2} t_{1}^{2}+h^{2} t_{3}^{2}+k^{2} t_{4}^{2}\right)}{32 \alpha_{1} \alpha_{2}}-\frac{b t_{2}\left(e t_{1}+h t_{3}+k t_{4}\right)}{\alpha_{1}}+\frac{e h t_{1} t_{3}+e k t_{1} t_{4}+h k t_{3} t_{4}}{32 \alpha_{1}} \\
& -\frac{c h}{2 \alpha_{1}} t_{3}^{2}-\frac{b h}{2 \alpha_{1}} t_{2}^{2}+\frac{e f}{64 \alpha_{1}} t_{1}^{2}+\frac{k m}{64 \alpha_{1}} t_{4}^{2}+\frac{h\left(e t_{1} t_{2}+f t_{1} t_{3}+k t_{2} t_{4}+m t_{3} t_{4}\right)}{64 \alpha_{1}}+\frac{\left(12 \alpha_{1}+\alpha_{2}\right) h^{2} t_{2} t_{3}}{32 \alpha_{1} \alpha_{2}} \\
& -\frac{d k}{2 \alpha_{1}} t_{4}^{2}-\frac{b k}{2 \alpha_{1}} t_{2}^{2}+\frac{e g}{64 \alpha_{1}} t_{1}^{2}+\frac{h m}{64 \alpha_{1}} t_{3}^{2}+\frac{k\left(e t_{1} t_{2}+g t_{1} t_{4}+h t_{2} t_{3}+m t_{3} t_{4}\right)}{64 \alpha_{1}}+\frac{\left(12 \alpha_{1}+\alpha_{2}\right) k^{2} t_{2} t_{4}}{32 \alpha_{1} \alpha_{2}} \\
& -\frac{c f}{2 \alpha_{1}} t_{3}^{2}-\frac{a f}{2 \alpha_{1}} t_{1}^{2}+\frac{e h}{64 \alpha_{1}} t_{2}^{2}+\frac{g m}{64 \alpha_{1}} t_{4}^{2}+\frac{f\left(e t_{1} t_{2}+g t_{1} t_{4}+h t_{2} t_{3}+m t_{3} t_{4}\right)}{64 \alpha_{1}}+\frac{\left(12 \alpha_{1}+\alpha_{2}\right) f^{2} t_{1} t_{3}}{32 \alpha_{1} \alpha_{2}} \\
& -\frac{c h}{2 \alpha_{1}} t_{3}^{2}-\frac{b h}{2 \alpha_{1}} t_{2}^{2}+\frac{e f}{64 \alpha_{1}} t_{1}^{2}+\frac{k m}{64 \alpha_{1}} t_{4}^{2}+\frac{h\left(e t_{1} t_{2}+f t_{1} t_{3}+k t_{2} t_{4}+m t_{3} t_{4}\right)}{64 \alpha_{1}}+\frac{\left(12 \alpha_{1}+\alpha_{2}\right) h^{2} t_{2} t_{3}}{32 \alpha_{1} \alpha_{2}} \\
& \frac{16 c^{2}}{\alpha_{1}} t_{3}^{2}+\frac{\left(12 \alpha_{1}+\alpha_{2}\right)\left(f^{2} t_{1}^{2}+h^{2} t_{2}^{2}+m^{2} t_{4}^{2}\right)}{32 \alpha_{1} \alpha_{2}}-\frac{c t_{3}\left(f t_{1}+h t_{2}+m t_{4}\right)}{\alpha_{1}}+\frac{f h t_{1} t_{2}+f m t_{1} t_{4}+h m t_{2} t_{4}}{32 \alpha_{1}} \\
& -\frac{d m}{2 \alpha_{1}} t_{4}^{2}-\frac{c m}{2 \alpha_{1}} t_{3}^{2}+\frac{f g}{64 \alpha_{1}} t_{1}^{2}+\frac{h k}{64 \alpha_{1}} t_{2}^{2}+\frac{m\left(f t_{1} t_{3}+g t_{1} t_{4}+h t_{2} t_{3}+k t_{2} t_{4}\right)}{64 \alpha_{1}}+\frac{\left(12 \alpha_{1}+\alpha_{2}\right) m^{2} t_{3} t_{4}}{32 \alpha_{1} \alpha_{2}} \\
& \left.-\frac{d g}{2 \alpha_{1}} t_{4}^{2}-\frac{a g}{2 \alpha_{1}} t_{1}^{2}+\frac{e k}{64 \alpha_{1}} t_{2}^{2}+\frac{f m}{64 \alpha_{1}} t_{3}^{2}+\frac{g\left(e t_{1} t_{2}+f t_{1} t_{3}+k t_{2} t_{4}+m t_{3} t_{4}\right)}{64 \alpha_{1}}+\frac{\left(12 \alpha_{1}+\alpha_{2}\right) g^{2} t_{1} t_{4}}{32 \alpha_{1} \alpha_{2}}\right) \\
& -\frac{d k}{2 \alpha_{1}} t_{4}^{2}-\frac{b k}{2 \alpha_{1}} t_{2}^{2}+\frac{e g}{64 \alpha_{1}} t_{1}^{2}+\frac{h m}{64 \alpha_{1}} t_{3}^{2}+\frac{k\left(e t_{1} t_{2}+g t_{1} t_{4}+h t_{2} t_{3}+m t_{3} t_{4}\right)}{64 \alpha_{1}}+\frac{\left(12 \alpha_{1}+\alpha_{2}\right) k^{2} t_{2} t_{4}}{32 \alpha_{1} \alpha_{2}} \\
& -\frac{d m}{2 \alpha_{1}} t_{4}^{2}-\frac{c m}{2 \alpha_{1}} t_{3}^{2}+\frac{f g}{64 \alpha_{1}} t_{1}^{2}+\frac{h k}{64 \alpha_{1}} t_{2}^{2}+\frac{m\left(f t_{1} t_{3}+g t_{1} t_{4}+h t_{2} t_{3}+k t_{2} t_{4}\right)}{64 \alpha_{1}}+\frac{\left(12 \alpha_{1}+\alpha_{2}\right) m^{2} t_{3} t_{4}}{32 \alpha_{1} \alpha_{2}} \\
& \left.\frac{16 d^{2}}{\alpha_{1}} t_{4}^{2}+\frac{\left(12 \alpha_{1}+\alpha_{2}\right)\left(g^{2} t_{1}^{2}+k^{2} t_{2}^{2}+m^{2} t_{3}^{2}\right)}{32 \alpha_{1} \alpha_{2}}-\frac{d t_{4}\left(g t_{1}+k t_{2}+m t_{3}\right)}{\alpha_{1}}+\frac{g k t_{1} t_{2}+g m t_{1} t_{3}+k m t_{2} t_{3}}{32 \alpha_{1}}\right)
\end{aligned}
$$

which after using the coordinates of the support points for the four ingredients design simplified to the matrix; 


$$
\begin{aligned}
& \left(\frac{103 a^{2}}{3 \alpha_{1}}+\frac{103\left(12 \alpha_{1}+\alpha_{2}\right)\left(e^{2}+f^{2}+g^{2}\right)}{1536}-\frac{77 a(e+f+g)}{144 \alpha_{1}}+\frac{77(e f+e g+f g)}{4608 \alpha_{1}}\right. \\
& -\frac{103 e(b+a)}{972 \alpha_{1}}+\frac{103(f h+g k)}{3072 \alpha_{1}}+\frac{77\left(12 \alpha_{1}+\alpha_{2}\right) e^{2}}{4608 \alpha_{1} \alpha_{2}}+\frac{77 e(f+g+h+k)}{9216 \alpha_{1}} \\
& C= \\
& -\frac{103 f(c+a)}{972 \alpha_{1}}+\frac{103(e h+g m)}{3072 \alpha_{1}}+\frac{77\left(12 \alpha_{1}+\alpha_{2}\right) f^{2}}{4608 \alpha_{1} \alpha_{2}}+\frac{77 f(e+g+h+m)}{9216 \alpha_{1}} \\
& -\frac{103 g(d+a)}{972 \alpha_{1}}+\frac{103(e k+f m)}{3072 \alpha_{1}}+\frac{77\left(12 \alpha_{1}+\alpha_{2}\right) g^{2}}{4608 \alpha_{1} \alpha_{2}}+\frac{77 g(e+f+k+m)}{9216 \alpha_{1}} \\
& -\frac{103 e(b+a)}{972 \alpha_{1}}+\frac{103(f h+g k)}{3072 \alpha_{1}}+\frac{77\left(12 \alpha_{1}+\alpha_{2}\right) e^{2}}{4608 \alpha_{1} \alpha_{2}}+\frac{77 e(f+g+h+k)}{9216 \alpha_{1}} \\
& \frac{103 b^{2}}{3 \alpha_{1}}+\frac{103\left(12 \alpha_{1}+\alpha_{2}\right)\left(e^{2}+h^{2}+k^{2}\right)}{1536}-\frac{77 b(e+h+k)}{144 \alpha_{1}}+\frac{77(e h+k g+h k)}{4608 \alpha_{1}} \\
& -\frac{103 h(c+b)}{972 \alpha_{1}}+\frac{103(e f+k m)}{3072 \alpha_{1}}+\frac{77\left(12 \alpha_{1}+\alpha_{2}\right) h^{2}}{4608 \alpha_{1} \alpha_{2}}+\frac{77 h(e+f+k+m)}{9216 \alpha_{1}} \\
& -\frac{103 k(d+b)}{972 \alpha_{1}}+\frac{103(e g+h m)}{3072 \alpha_{1}}+\frac{77\left(12 \alpha_{1}+\alpha_{2}\right) k^{2}}{4608 \alpha_{1} \alpha_{2}}+\frac{77 k(e+g+h+m)}{9216 \alpha_{1}} \\
& -\frac{103 f(c+a)}{972 \alpha_{1}}+\frac{103(e h+g m)}{3072 \alpha_{1}}+\frac{77\left(12 \alpha_{1}+\alpha_{2}\right) f^{2}}{4608 \alpha_{1} \alpha_{2}}+\frac{77 f(e+g+h+m)}{9216 \alpha_{1}} \\
& -\frac{103 h(c+b)}{972 \alpha_{1}}+\frac{103(e f+k m)}{3072 \alpha_{1}}+\frac{77\left(12 \alpha_{1}+\alpha_{2}\right) h^{2}}{4608 \alpha_{1} \alpha_{2}}+\frac{77 h(e+f+k+m)}{9216 \alpha_{1}} \\
& \frac{103 c^{2}}{3 \alpha_{1}}+\frac{103\left(12 \alpha_{1}+\alpha_{2}\right)\left(f^{2}+h^{2}+m^{2}\right)}{1536}-\frac{77 c(f+h+m)}{144 \alpha_{1}}+\frac{77(f h+f m+h m)}{4608 \alpha_{1}} \\
& -\frac{103 m(c+d)}{972 \alpha_{1}}+\frac{103(f g+h k)}{3072 \alpha_{1}}+\frac{77\left(12 \alpha_{1}+\alpha_{2}\right) m^{2}}{4608 \alpha_{1} \alpha_{2}}+\frac{77 m(f+g+h+k)}{9216 \alpha_{1}} \\
& -\frac{103 g(d+a)}{972 \alpha_{1}}+\frac{103(e k+f m)}{3072 \alpha_{1}}+\frac{77\left(12 \alpha_{1}+\alpha_{2}\right) g^{2}}{4608 \alpha_{1} \alpha_{2}}+\frac{77 g(e+f+k+m)}{9216 \alpha_{1}} \\
& -\frac{103 k(d+b)}{972 \alpha_{1}}+\frac{103(e g+h m)}{3072 \alpha_{1}}+\frac{77\left(12 \alpha_{1}+\alpha_{2}\right) k^{2}}{4608 \alpha_{1} \alpha_{2}}+\frac{77 k(e+g+h+m)}{9216 \alpha_{1}} \\
& -\frac{103 m(c+d)}{972 \alpha_{1}}+\frac{103(f g+h k)}{3072 \alpha_{1}}+\frac{77\left(12 \alpha_{1}+\alpha_{2}\right) m^{2}}{4608 \alpha_{1}}+\frac{77 m(f+g+h+k)}{9216 \alpha_{1}} \\
& \left.\frac{103 d^{2}}{3 \alpha_{1}}+\frac{103\left(12 \alpha_{1}+\alpha_{2}\right)\left(g^{2}+k^{2}+m^{2}\right)}{1536}-\frac{77 d(g+k+m)}{144 \alpha_{1}}+\frac{77(g k+g m+k m)}{4608 \alpha_{1}}\right)
\end{aligned}
$$

With

$$
\begin{aligned}
\text { traceC }= & \frac{103\left(a^{2}+b^{2}+c^{2}+d^{2}\right)}{3 \alpha_{1}}+\frac{103\left(12 \alpha_{1}+\alpha_{2}\right)\left(e^{2}+f^{2}+g^{2}+h^{2}+k^{2}+m^{2}\right)}{768} \\
& -\frac{77(a e+a f+a g+b e+b h+b k+c f+c h+c m+d g+d k+d m)}{486 \alpha_{1}} \\
& +\frac{77(e f+e g+f g+e h+e k+h k+f h+f m+h m+g k+g m+k m)}{243 \alpha_{1} \alpha_{2}} \\
= & 2.2545 \times 10^{4}
\end{aligned}
$$

where the A-optimal slope weight vector entries were employed and coefficient values from the fitted model.

The A-optimal slope information was gotten by using equation (10) with $p=-1, m=4$ and the trace value from (23) as;

$$
v\left(\phi_{-1}\right)=\left(\frac{1}{4} \text { traceC }\right)^{-1}=\left(\frac{2.2545 \times 10^{4}}{4}\right)^{-1}=1.7742 \times 10^{-4} .
$$

\section{Conclusion}

The design presented is highly efficient for designs with fixed sample size. As is clear from the fruit blending experiment, the designs describe the response surface involved very well. However, the experimenter is mandated to ensure accuracy in the measurement of ingredient amounts and to determine the necessary number of replications required to achieve the desired precision levels.

\section{References}

Aggarwal, M. L., Singh, P., Sarin, V., \& Husain, B. (2013). Optimal Orthogonal Block Designs for Four-Mixture Components in Two Blocks Based on F-squares for Becker's Models and K-model. Journal of Theoretical and 
Applied Statistics, 47(5), 1003-1021. https://doi.org/10.1080/02331888.2012.667102

Draper, N. R., \& Pukelsheim, F. (1998). Mixture Models Based on Homogeneous Polynomials. Journal of statistical planning and inference, 71, 303-311. https://doi.org/10.1016/S0378-3758(98)00012-3

Draper, N. R., Heiligers, B., \& Pukelsheim, F. (2000). Kiefer Ordering of Simplex Designs for Mixture Models with Four Ingredients. Annals of Statistics, 28, 578-235-257. https://doi.org/10.1214/aos/1016218231

Klein, T. (2004). Invariant Symmetric Block Matrices for the Design of Mixture Experiments. Linear Algebra and its Applications, 388, 261-278. https://doi.org/10.1016/S0024-3795(03)00486-5

Klein, T. (2004). Optimal Designs for Second-Degree Kronecker Model Mixture Experiments. Journal of Statistical Planning and Inference, 123, 117-131. https://doi.org/10.1016/S0378-3758(03)00145-9

Murty, N. N., \& Studden, W. J. (1972). Optimal Designs for Estimating Slope of a Polynomial Regression. Journal of America Statistics Association, 67, 869-873. https://doi.org/10.1080/01621459.1972.10481308

Ott, L. S., \& Mendenhall, W. (1972). Designs for Estimating the Slope of a Second Order Linear Model. Technometrics, (14), 341-353. https://doi.org/10.1080/00401706.1972.10488920

Pukelsheim, F. (1993). Optimal Designs of Experiments. New york: wiley.

Sceffe', H. (1958). Experiments with Mixtures. J. Roy. Statist. Ser., $\quad$ (B20), $344-360$. https://doi.org/10.1111/j.2517-6161.1958.tb00299.x

Sheffe', H. (1963). The Simplex-Centroid Design for Experiments with Mixtures. J. Roy. Statist. Soc. Ser., (25), $235-257$. https://doi.org/10.1111/j.2517-6161.1963.tb00506.x

Sung, H. P., Hyang, S. J., \& Rabindra, N. D. (2009). Slope-Rotatability of Second Order Response Surface Regression Models with Corrected Error. Quality Technology and Quantitative Management, 6(4), 471-492. https://doi.org/10.1080/16843703.2009.11673211

\section{Copyrights}

Copyright for this article is retained by the author(s), with first publication rights granted to the journal.

This is an open-access article distributed under the terms and conditions of the Creative Commons Attribution license (http://creativecommons.org/licenses/by/4.0/). 